\title{
Immune regulation of a chronic bacteria infection and consequences for pathogen transmission
}

\author{
Ashutosh K Pathak' ${ }^{1}$ Kathleen E Creppage ${ }^{1}$, Jacob R Werner ${ }^{2}$, Isabella M Cattadori ${ }^{*}$
}

\begin{abstract}
Background: The role of host immunity has been recognized as not only playing a fundamental role in the interaction between the host and pathogen but also in influencing host infectiousness and the ability to shed pathogens. Despite the interest in this area of study, and the development of theoretical work on the immunoepidemiology of infections, little is known about the immunological processes that influence pathogen shedding patterns.

Results: We used the respiratory bacterium Bordetella bronchiseptica and its common natural host, the rabbit, to examine the intensity and duration of oro-nasal bacteria shedding in relation to changes in the level of serum antibodies, blood cells, cytokine expression and number of bacteria colonies in the respiratory tract. Findings show that infected rabbits shed B. bronchiseptica by contact up to 4.5 months post infection. Shedding was positively affected by number of bacteria in the nasal cavity (CFU/g) but negatively influenced by serum IgG, which also contributed to the initial reduction of bacteria in the nasal cavity. Three main patterns of shedding were identified: i- bacteria were shed intermittently (46\% of individuals), ii- bacteria shedding fell with the progression of the infection (31\%) and iii- individuals never shed bacteria despite being infected (23\%). Differences in the initial number of bacteria shed between the first two groups were associated with differences in the level of serum antibodies and white blood cells. These results suggest that the immunological conditions at the early stage of the infection may play a role in modulating the long term dynamics of B. bronchiseptica shedding.

Conclusions: We propose that lgG influences the threshold of bacteria in the oro-nasal cavity which then affects the intensity and duration of individual shedding. In addition, we suggest that a threshold level of infection is required for shedding, below this value individuals never shed bacteria despite being infected. The mechanisms regulating these interactions are still obscure and more studies are needed to understand the persistence of bacteria in the upper respiratory tract and the processes controlling the intensity and duration of shedding.
\end{abstract}

\section{Background}

An appreciation of the immunological mechanisms that affect the interaction between the host and its pathogens is crucial for an understanding of the epidemiology of infection [1-4]. By linking within-host immunological processes to the between-host dynamics of infection it is possible to explain, and ultimately prevent, the conditions that allow for the invasion and survival of a pathogen within a host and the consequences for transmission. Fundamental to this is the knowledge of how the immune response affects pathogen replication and

\footnotetext{
* Correspondence: imc3@psu.edu

'Center for Infectious Disease Dynamics, Dept. Biology, The Pennsylvania

State University, University Park PA 16802, USA

Full list of author information is available at the end of the article
}

clearance as well as the intensity and duration of shedding and, thus, transmission.

Chronic bacteria infections can pose a challenge to the study of host infectiousness and associated immune response in that bacteria can either persist in the host, despite an acute inflammatory phase and active immunity, or colonize and persist without causing any apparent clinical or symptomatic effects [5-7]. Bacteria can activate their pathogenicity at a later time by triggering serious disease and high infectiousness or can increase their transmission rate in response to changes in host susceptibility [8-12]. These findings suggest that immune-compromised and chronically infected hosts can act either as life-long bacteria shedders or shed bacteria for a restricted period, usually coinciding with the 
acute phase of infection. To understand the dynamics of chronic infections, we need to identify not only the key immunological processes that affect long term pathogen persistence but also how pathogen replication, intensity and duration of bacteria shedding is associated with the immune response.

Here, we investigated the relationship between immune response and shedding rate in a chronic bacteria infection using the Bordetella bronchiseptica-rabbit system. Our recent work on the epidemiology of B. bronchiseptica in a free living population of rabbits (Oryctolagus cuniculus) showed that this is a common and persistent infection: annual prevalence ranged between $88 \%$ and $97 \%$ and by 2 months of age, $65 \%$ of the individuals had already seroconverted [13]. A model for bacteria infection was suggested where the annual recruitment of new infected individuals was associated with the onset of the host breeding season and the availability of new naïve offspring. Breeding, seropositive females represented the main source of infection for the newborns. However, it was not clear whether they were chronically infectious or in a re-activated infectious status due to the immuno-suppressed conditions during breeding.

Current knowledge on the immunology of B. bronchiseptica infection is largely derived from laboratory work with rats and mice and occasionally rabbits [14-21]. Studies on mice suggest that the bacterium stimulates an initial strong innate and subsequent acquired immune response characterized by the clearance of the bacteria from the lower respiratory tract but the persistence in the nasal cavity up to 270 days post infection, with the potential for life-long bacteria shedding [15]. The mechanisms involved in the persistence of bacteria in the nasal cavity are still unclear but the adhesin filamentous hemagglutinin (FHA) appears to play an important role in the colonization of the unciliated olfactory epithelia [22]. While highly informative, rats and mice show no documented ability for oro-nasal B. bronchispetica transmission and are not useful hosts for exploring the effect of host immunity on bacteria shedding and transmission in general $[23,24]$.

Motivated by our recent work on the epidemiology of $B$. brochiseptica infection in a natural system, we examined whether chronically infected individuals can be long-term, constant bacteria shedders or whether the frequency and intensity of shedding changes with time and between individuals as constrained by their immune response; for example, hosts may not shed bacteria despite being chronically infected. We established a laboratory model system wherein rabbits were infected with B. bronchiseptica strain RB50 and acquired immunity and bacteria shedding was quantified for 150 days post infection. We focused our attention on the immunological parameters relevant to the dynamics of B. bronchiseptica, as previously identified in mice and rabbits, and examined how they affect the intensity and duration of the oro-nasal shedding.

\section{Results}

To highlight heterogeneities in the shedding pattern and associated immune response between individuals, blood and tissue samples were individually processed.

\section{Infection of rabbits with $B$. bronchiseptica RB50}

Intranasal infection of rabbits led to the successful colonization and establishment of bacteria in the entire respiratory tract. By 3 days post infection (DPI) the mean number of bacteria colonies in the respiratory tract was 232 times higher than the initial inoculum $(50,000 \mathrm{CFU} /$ $\mathrm{ml}$, Fig. 1). Levels peaked at day 7 post infection in all the three organs but quickly decreased thereafter and, by 150 days post infection, $B$. bronchiseptica was completely cleared from the trachea and lungs but persisted in the nares (Fig. 1). The number of bacteria consistently declined with the duration of the infection, DPI (coeff \pm S.E.: $-0.111 \pm 0.013$ d.f. $=30, \mathrm{P}<0.0001$ ) but nares were significantly higher than either trachea or lungs

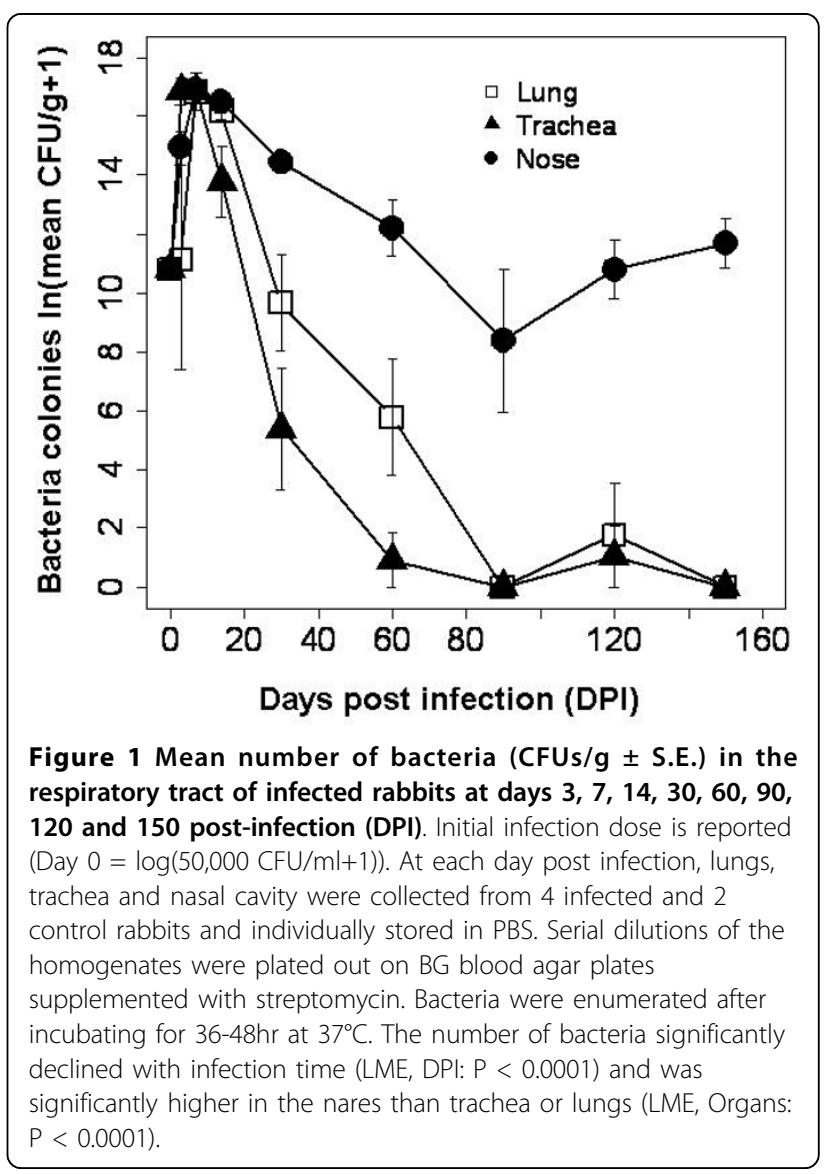


(coeff \pm S.E.: $0.069 \pm 0.017$ d.f. $=60 \mathrm{P}<0.0001$ ), once differences among individuals and the non-independent sampling of the three organs from the same host were taken into account. Number of bacteria in the respiratory tract was negatively affected by serum IgG and circulating lymphocytes (coeff. \pm S.E.: $-6.5714 \pm 1.002$ and $-0.853 \pm$ 0.306 , respectively) but positively influenced by circulating neutrophils (coeff. \pm S.E.: $1.709 \pm 0.524$ ), when corrected by host variability and the non-independence of sampling the three respiratory organs from the same individual (For all: d.f. $=23, \mathrm{P}<0.01)$. The analysis repeated for each organ confirmed the negative effect of IgG on bacteria in the nares (coeff \pm S.E.: $-4.221 \pm 0.854$, d.f $=30, \mathrm{P}<0.0001$ ) but also highlighted the positive effect of IL-10 (coeff \pm S.E: $-4.210 \pm 0.512$ ) and the negative role of IL-4 (coeff \pm S.E: $3.431 \pm 0.748$ ) on bacteria in the lungs (analysis based on $\mathrm{Ct}$ values, for both: d.f. $=28, \mathrm{P}<0.0001)$. It is important to note that the cycle threshold $(\mathrm{Ct})$ is inversely related to cytokine expression level, therefore and as reported above, the sign of the coefficient describing the CFU-Ct relationship should be interpreted as positive when negative and vice-versa. Results also showed a negative effect of serum antibodies and circulating lymphocytes (IgG, IgA and lymphocytes coeff \pm S.E.: $-9.564 \pm 1.225$, $-5.046 \pm 1.769$ and $-1.006 \pm 0.372$, respectively) and a positive effect of circulating neutrophils (coeff \pm S.E.: $2.168 \pm$ 0.636 ) on bacteria in the trachea (for all: d.f. $=22, \mathrm{P}<$ $0.01)$. Overall, these findings support the hypothesis that IgG, IgA, neutrophils and lymphocytes are heavily involved in B. bronchiseptica clearance from the lower but not the upper respiratory tract, despite the negative effect of IgG. The positive association with neutrophils is probably caused by their rapid recruitment and short-lived contribution in the bacteria removal, as previously recorded [15,25]. Moreover, our results further support the suggestion of an immunological interference between antibody-mediated clearance (mainly by IgG) and antagonistic IL-10 anti-inflammatory activity in the lungs, which may explain the delay in bacteria clearance from this site as reported in other models [17].

\section{Bacteria shedding}

The goal of this study was to quantify the rate of $B$. bronchiseptica shedding in relation to the immune response and to use this finding to gain stronger insights into the epidemiology of a chronic infection. The strain of B. bronchiseptica used in this work was originally isolated from the nares of a 3 month old New Zealand White rabbit and it was assumed that it could be naturally transmitted between individuals [14]. Indeed, we found that rabbits were able to shed bacteria onto a BG blood agar plate by direct oro-nasal contact, which mimicked the natural contacts observed between free living individuals. Mean number of bacteria shed per second was 0.028 ( \pm 0.001 S.E.) CFUs; shedding was high during the first month post infection and again 15 weeks later but substantially dropped between the two peaks (Fig. 2). Based on the longitudinal data (weekly sampling of individuals for serum antibodies and blood cells), we found a significant negative effect of IgG on number of bacteria shed (coeff \pm S.E: $-0.092 \pm 0.025 \mathrm{df}=88 \mathrm{P}<0.0001$ ), once corrected by host variability. Blood cells did not contribute to the pattern observed. The analysis was repeated using bacteria CFU counts from the nares of animals sampled at $60,90,120$ and 150 post infection, and a weak but significant positive relationship was observed between bacteria shed at these sampling points and bacteria in the nasal cavity (coeff \pm S.E.: $0.37 \mathrm{e}-7 \pm 0.14 \mathrm{e}-7$ d.f. $=8 \mathrm{P}<0.030)$. Together these results suggest that shedding is positively influenced by the level of infection in the oro-nasal cavity and negatively affected by serum IgG.

Three main patterns of shedding were identified during the course of the infection: i- bacteria were shed

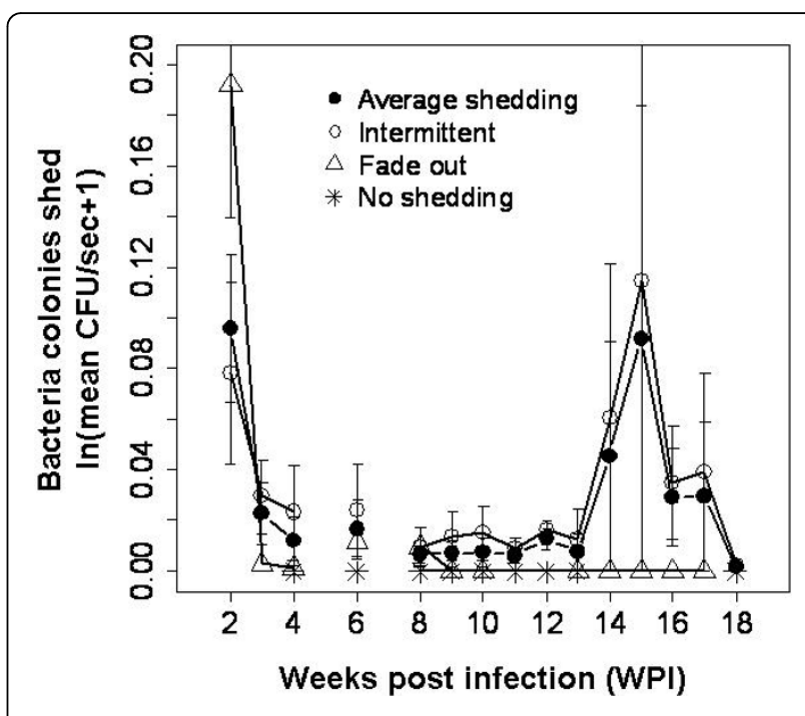

Figure 2 Mean number of bacteria shed (CFUs/sec \pm S.E.) by oro-nasal contact with a BG blood agar plate during the course of the infection. A total of 14 infected rabbits were used and sacrificed at days 60, 90, 120 and 150 post-infection. Each individual was weekly challenged by oro-nasal contact with a BG blood agar plate and time of interaction measured. Bacteria were enumerated after incubating for $36-48 \mathrm{hr}$ at $37^{\circ} \mathrm{C}$. For every week post infection (from WPI 2 to WPI 18) the number of plates positive for $B$. bronchiseptica after removal of contaminated cases and sacrificed individuals was: WPI $2=8$, WPI $3=6$, WPI $4=8$, WPI $5=$ N.D. (no data), WPI $6=11$, WPI $7=$ N.D., WPI $8=14$, WPI $9=12$, WPI $10=12$, WPI $11=12$, WPI $12=12$, WPI $13=8$, WPI $14=8$, WPI $15=8$, WPI $16=8$, WPI $17=8$, WPI $18=4$. The overall average shedding pattern and the more specific three shedding groups (intermittent, fade-out and non-shedding) are reported. 
with variable intensities at irregular intervals ('intermittent' group, $46 \%$ of individuals), ii- intensity of bacteria shed fell with the progression of the infection ('fade-out' group, $31 \%$ ) and iii- individuals never shed bacteria despite being infected ('non-shedders', 23\%) (Fig. 2). To highlight immunological differences associated with these shedding patterns, analyses were repeated among the three groups using longitudinal blood data. No significant differences in serum IgG, IgA, neutrophils and lymphocytes were observed among the three patterns, however, the intercept of the models was consistently significant (for all: $\mathrm{P}<0.05$ ), once corrected for variability between hosts and their multiple sampling. This finding supports the hypothesis that the strength of the initial immune response is crucial in modulating the dynamics of shedding. During the second week post infection, differences in the dynamics of infection were observed between the intermittent and the fade-out group (no data were available for the non-shedding group). The relatively low number of bacteria shed by the intermittent group (mean CFU/sec. \pm S.E.: $0.083 \pm$ 0.019 ) was associated with low serum IgG (OD index \pm S.E.: $0.238 \pm 0.028)$ and high serum IgA $(1.107 \pm 0.052)$ as well as high circulating neutrophils (mean $\mathrm{K} / \mu \mathrm{L} \pm$ S.E.: $1.436 \pm 0.158$ ) and lymphocytes (mean $\mathrm{K} / \mu \mathrm{L} \pm$ S.E.: $2.150 \pm 0.412)$. In contrast, the higher shedding in the fade out group (mean CFU/sec. \pm S.E.: $0.213 \pm 0.045$ ) was correlated to high serum IgG (OD index \pm S.E.: $0.434 \pm 0.118)$ and low serum IgA $(0.667 \pm 0.128)$ and white blood cells (mean $\mathrm{K} / \mu \mathrm{L} \pm$ S.E., neutrophils: $0.896 \pm 0.00$ and lymphocytes: $0.740 \pm 0.000$ ). Although not conclusive or statistically significant, these relationships suggest that the strength of the early antibody and blood cells response may play a role in affecting both the initial and long-term pattern of $B$. bronchiseptica transmission.

\section{Host immune response overview}

Overall, the immune response of rabbits to B. bronchiseptica infection confirmed previous findings reported in other animal models [14-19,25].

\section{Peripheral response}

Infected hosts developed a strong serum IgG and IgA response compared to the controls (Fig. 3). The level of IgG rapidly increased in infected rabbits and remained consistently high for the duration of the infection, however and as previously highlighted, it was not sufficient to completely clear the bacteria from the upper respiratory tract (interaction between sampling time and infected-controls, coeff \pm S.E.: $0.047 \pm 0.005$ d.f. $=328 \mathrm{P}$ $<0.0001$-corrected for the random effect of the host and its longitudinal sampling). IgA levels in infected rabbits peaked around week three post infection and

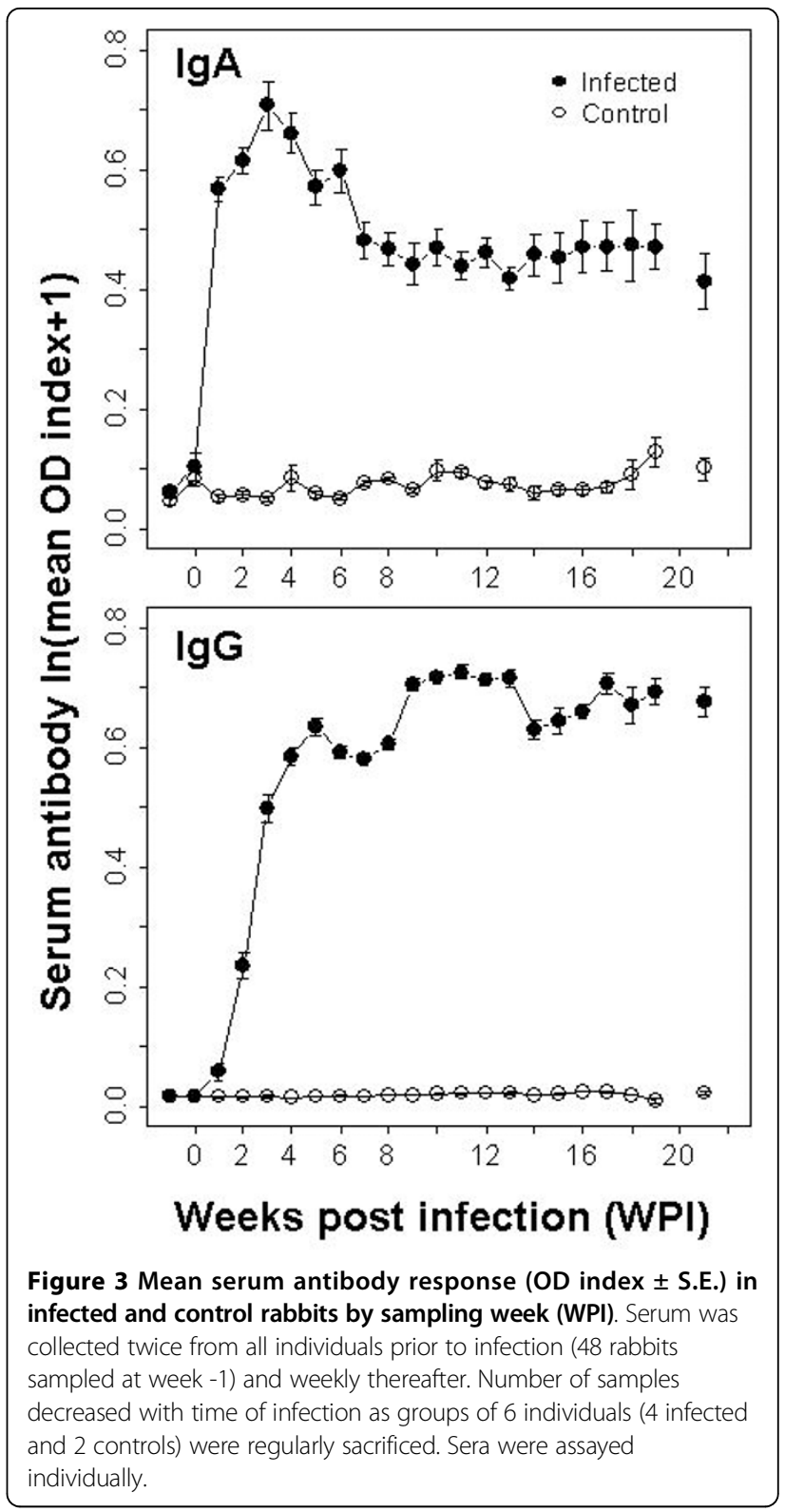

decreased thereafter, probably as a consequence of the successful clearance of bacteria from the lower respiratory tract $[25,26]$. Nevertheless, values remained significantly higher in infected compared to controls (coeff \pm S.E.: $0.208 \pm 0.056$ d.f. $=45 \mathrm{P}<0.001)$ and for the duration of the experiment (interaction between infectedcontrols and sampling time, coeff \pm S.E.: $0.0026 \pm 0.001$ d.f. $=410 \mathrm{P}<0.01$; corrected for the host variability). Collectively, the systemic antibody profiles suggest that rabbit immune protection against $B$. bronchiseptica is robust for the first 5 months (150 days) post infection but does not result in complete clearance.

The neutrophil concentration in the blood decreased with the duration of the infection (coeff \pm S.E.:- $0.011 \pm$ 0.002 d.f $=334 \mathrm{P}<0.0001)$ and was similar between 
infected and controls except in the first 2 weeks post-infection, where a significant neutrophilia was observed in infected compared to controls (coeff \pm S.E.: $0.159 \pm 0.075$ d.f. $=27 \mathrm{P}<0.05)$. These findings further support the short-lived and early involvement of neutrophils in B. bronchiseptica clearance $[15,27]$.

\section{Cytokine response in the lungs}

As shown in fig. 4 and based on the $2^{-\Delta \Delta c t}$ transformation, a high IL-10 expression was observed in the lungs of infected rabbits in the first 30 days post infection, this was followed by a short-lived peak in IFN- $\gamma$ at 60 days post infection, and a general decrease in cytokine expression thereafter. IL-4 showed consistent baseline expression. Overall and using the raw $\mathrm{Ct}$ values for analysis tractability, results confirmed the important anti-inflammatory role of IL-10 in B. bronchiseptica infected rabbits (interaction between infected-controls and sampling time, coeff \pm S.E.: $0.001 \pm 0.0001$ d.f. $=41 \mathrm{P}<0.05$, corrected for the random effect of the host). IFN- $\gamma$ and IL-4 $\mathrm{Ct}$ values significantly changed among sampling time but not between infected and controls (respectively, coeff \pm S.E.: $0.001 \pm 0.0003$ and $-0.001 \pm 0.0003$ for both d.f. $=$ $42 \mathrm{P}<0.05)$. Through its anti-inflammatory properties and involvement in the recruitment and activation of other anti-inflammatory cells $[28,29]$, IL-10 probably facilitated the establishment of bacteria in the respiratory tract and the subsequent persistence in the nares, while the peaks at 7 and 60 days post infection in IFN- $\gamma$

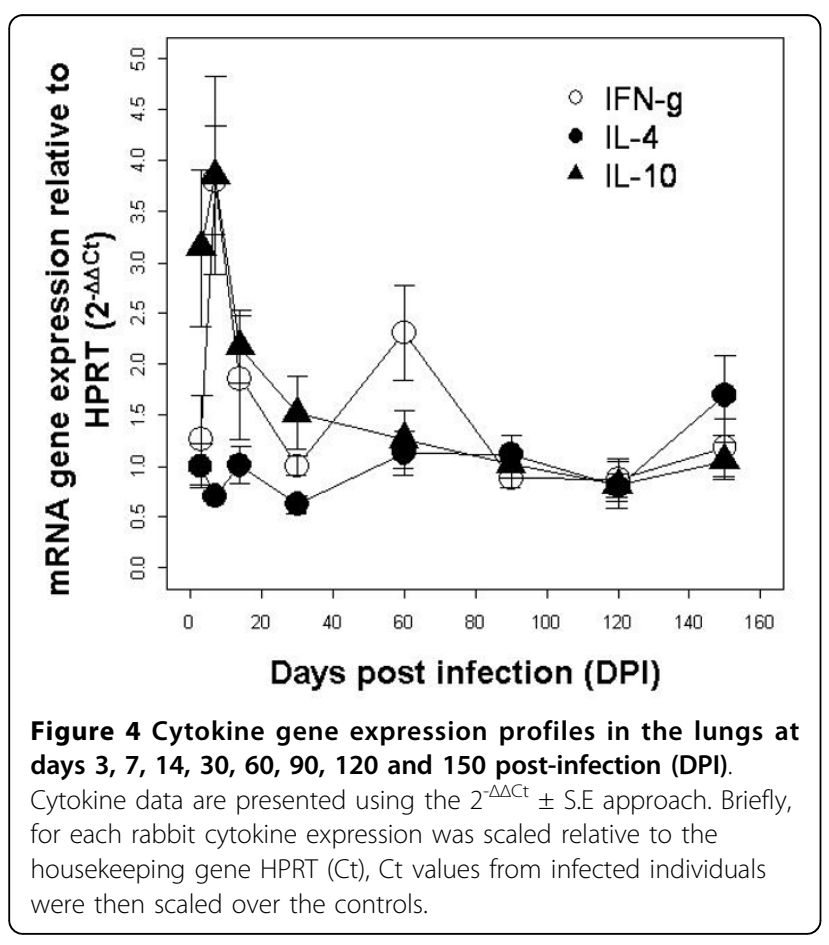

confirmed its important role in bacteria clearance from the lungs and possibly trachea. In summary, the dynamics of cytokine expression in the lungs of infected rabbits was in line with previous studies $[20,21]$.

\section{Discussion}

This study showed that rabbits infected with Bordetella bronchiseptica strain RB50 were able to shed bacteria by oro-nasal contact for at least 128 days post infection. Individuals naturally interacted with BG blood agar plates and a few minutes of direct contact were sufficient for the transmission of bacteria onto the medium. The mean number of bacteria shed followed the dynamics of infection, in that, shedding was high during the initial first month and decreased thereafter, although occasional peaks were observed up to 17 weeks post infection. The variability in the shedding pattern was unexpected but supports the hypothesis that rabbits with a chronic $B$. bronchiseptica infection can be long-term shedders, through a persistent infection in the upper respiratory tract. Specifically, most of the bacteria were shed at irregular intervals and with intensities that vary both within and between individuals. However, we also showed that some individuals never shed bacteria while infected, and this supports the hypothesis of a non-linear relationship between host infectiousness and B. bronchiseptica transmission. Moreover, since the immune system imposed constrains on the level and duration of infection we may argue that there was also a non-linear relationship between immune response and transmission dynamics.

The host acquired immunity, and probably the level of the early response, influenced the intensity, duration and pattern of bacteria shed. Serum IgG appeared to contribute to bacteria clearance in the lungs and trachea and the initial reduction in the nares. IgG also exerted a negative effect on the amount of B. bronchiseptica shed and together with IgA and white blood cells appeared to influence the initial and long-term shedding pattern. Indeed, a robust and timely IgG response probably modulated the long term shedding of $B$. bronchiseptica by quickly reducing or controlling replication in the nares below a threshold value required for consistent and prolonged pathogen transmission. In contrast, it is possible that the initial lower infection levels stimulated a milder immune response that allowed bacteria replication above a threshold necessary for long term shedding. While the number of bacteria in the nares was positively associated to the level of bacteria shed, some infected individuals never shed bacteria, supporting the hypothesis that a minimum threshold level of infection is necessary for bacteria shedding. 
Serum IgA was probably more involved in the initial clearance of the lower respiratory tract, which agrees with the general role of this immunoglobulin in the early protection against invasive infections [26]. Serum IgG and IgA have been previously shown to be sufficient for B. bronchiseptica clearance in the lower but not the upper respiratory tract [16-18,25]. Similarly, neutrophils are involved in the early clearance of $B$. bronchiseptica from the lower respiratory tract $[16,26,30]$. Our findings on the role of serum antibodies and bacteria clearance are in line with previous work but also highlight the effect of serum IgG on the dynamics of B. bronchiseptica shedding.

At the cytokine level, the high IL-10 in the lungs during the initial 2 months of infection, was fundamental to delay clearance in the lower respiratory tract and probably contributed to the persistence of bacteria in the nasal cavity and associated shedding. Indeed, the absence of IL-10 synthesis has been related to augmented $B$. bronchiseptica clearance as well as reduced, albeit more effective, antibody production and higher IFN- $\gamma$ in mice [17]. The association between serum antibodies, cytokines and bacteria shed has been reported in other host-bacteria systems. For example, a negative relationship between fecal shedding of Escherichia coli O157:H7 and IgG and IgA was observed in cows previously infected with a homologous bacteria strain [31]. Mucosal IgA was shown to reduce vaginal shedding and reinfection with C. trachomatis in mice [32], while human infections with Campylobacter spp. exhibited an inverse relationship between the shedding of fecal bacteria and age-dependent increases in serum IgG and IgA [33]. Moreover, IFN- $\gamma$ expression appeared to contribute to the reduction of Chlamydia trachomatis and C. muridarum shedding in mice [34,35].

\section{Conclusions}

We showed that rabbits were heterogeneous in their pattern of shedding B. bronchiseptica and that this was associated with differences in the host immune response. The dynamics of infection and partial clearance was consistent among individuals and a positive relationship was observed between bacteria shed and bacteria in the nasal cavity. Yet, some hosts shed bacteria intermittently, others shed bacteria only during the initial few weeks of infection while some individuals never shed bacteria. Together these findings suggest a strong non-linear relationship between force of infection, immune response and shedding rate for this chronic infection. The molecular mechanisms regulating these interactions are still obscure and more studies are needed to understand the persistence of bacteria in the upper respiratory tract as well as the processes controlling bacteria dispersal through direct oro-nasal contact or aerosol.

The occurrence of individuals that did not shed bacteria and the exclusion of a few contaminated plates, especially from the early part of the study, affected our search for a robust association between shedding patterns and the immune response. Nevertheless, the general patterns of bacteria dynamics and immune response, currently described, are consistent in this host-pathogen system as confirmed by our more recent studies on rabbits co-infected with B. bronchiseptica and gastrointestinal nematodes (unpubl. data). In conclusion, more attention should be given to the understanding of the relationship between host immune response, the level of infection and heterogeneities in pathogen shedding.

\section{Methods}

\section{Bacteria strain and culture}

The Bordetella bronchiseptica strain RB50 used in this study was kindly provided by Dr. E. T. Harvill (Penn State University, PA, USA). Bacteria were grown on Bordet-Gengou (BG) agar (HiMedia, USA) supplemented with $10 \%$ defibrinated sheep blood (Hema Resources, USA) and streptomycin $(20 \mu \mathrm{g} / \mathrm{ml}$, Sigma-Aldrich Co., USA). The bacteria inoculum was prepared by growing in Stainer-Scholte (SS) liquid culture medium at $37^{\circ} \mathrm{C}$ overnight on a rotary shaker to an optical density at 600 $\mathrm{nm}$ of approximately 0.3 . For the infection, bacteria were re-suspended in sterile phosphate-buffered saline (PBS) at a density of $5 \times 10^{4} \mathrm{CFUs} / \mathrm{ml}$, which was confirmed by plating serial dilutions of the inoculum on BG blood agar plates in triplicate.

\section{Study design}

Out-bred, 60 days old New Zealand White male rabbits free from B. bronchiseptica and other pathogens/parasites (Harlan, USA), were housed in individual cages with food and water ad libitum and a $12 \mathrm{~h}$ day/night cycle. Individuals were lightly sedated intravenously with a pre-mixed solution of Ketamine $(5 \mathrm{mg} / \mathrm{kg}$, Phoenix Pharmaceuticals, USA) and Valium $(0.25 \mathrm{mg} / \mathrm{kg}$, Hospira, USA) and intra-nasally infected by pipetting in each nare $0.5 \mathrm{ml}$ of PBS containing $2.5 \times 10^{4} \mathrm{~B}$. bronchiseptica (dose adapted from [14]). Control animals were sham inoculated with $1 \mathrm{ml}$ of sterile PBS. Groups of 6 individuals ( 4 infected and 2 controls) were euthanized with $1 \mathrm{ml}$ of pentobarbital (Euthasol, Virbac) at days 3, $7,14,30,60,90,120$ and 150 post-infection (DPI) and the lungs, trachea and nasal cavity removed aseptically. Blood samples were collected weekly from the marginal ear vein of all animals. Animals were weighed weekly and monitored routinely for health status. All listed 
animal procedures were pre-approved by the Institutional Animal Care and Use Committee of The Pennsylvania State University.

\section{Quantification of bacteria in the respiratory tract}

Following euthanasia, a weighed amount of the trachea and nasal cavity (turbinates and septum) were homogenized in 5 and $15 \mathrm{mls}$ of PBS, respectively. The lungs were blended and approximately $3 \mathrm{~g}$ of the mix transferred into tubes containing RNAlater (Qiagen) and stored at $-80^{\circ} \mathrm{C}$ for subsequent cytokine determination. The remaining tissue was homogenized in $15 \mathrm{ml}$ of sterile PBS. Serial dilutions of the tissue homogenates were plated onto BG blood agar plates and incubated at $37^{\circ} \mathrm{C}$ for 48 hours to allow bacteria quantification.

\section{Quantification of bacteria shed}

To monitor the weekly amount of bacteria shed, 14 infected rabbits were selected from the late sampling points $(60,90,120$ and 150 DPI). Every week, a BG blood agar plate was left in each cage for a maximum of 10 minutes and rabbits were allowed to interact with the plate by direct oral-nasal contact; the duration of each interaction was recorded. Plates were removed in case individuals chewed the plastic or ate the agar. Bacteria colonies were counted after incubation at $37^{\circ} \mathrm{C}$ for 48 hours; data were expressed as number of bacteria colonies shed per second of active interaction. This procedure is analogous to a natural transmission process compared to the nasal swabbing method that is more invasive, disruptive of the bacteria population and less representative of the individual's ability to shed bacteria [36-38]. We were not able to record the first week post infection data due to technical problems.

\section{Enzyme-Linked Immunosorbent Assay (ELISA)}

Serum was collected once a week from all animals and separated from the red blood cells by centrifugation $(10,000 \mathrm{rpm}$ for $10 \mathrm{~min})$ and stored at $-80^{\circ} \mathrm{C}$. Antigen coated plates were prepared by growing $B$. bronchiseptica overnight to mid-log phase in SS culture medium (OD at $600 \mathrm{~nm}$ of 0.6 ), washed once and re-suspended in PBS. Bacteria were heat inactivated at $65^{\circ} \mathrm{C}$ for 30 minutes, centrifuged at $5000 \mathrm{rpm}$ for 15 minutes at $4^{\circ} \mathrm{C}$ and the resulting lysate estimated for protein concentration with the BCA assay (Pierce Biotechnology). The lysate was diluted in $0.2 \mathrm{M}$ carbonate/bicarbonate coating buffer $(\mathrm{pH}$ 9.6) to obtain a final concentration of $6.5 \mu \mathrm{g} / \mathrm{ml} .100 \mu \mathrm{l}$ was used to coat the wells of 96well polystyrene plates (Greiner Bio-One). Plates were incubated overnight at $4{ }^{\circ} \mathrm{C}$ and then frozen at $-20^{\circ} \mathrm{C}$ until use. Prior to serum addition, the plates were thawed at $37^{\circ} \mathrm{C}$ for 1 hour and blocked in $5 \%$ non-fat milk and PBS-T for 1 hour. The optimal serum dilution for the IgA and IgG ELISA assays was performed following Sanchez et al. [39] and Crowther [40]. A pool from strongly reacting serum samples (high pool prepared from infected individuals 4-6 weeks post-infection) and a pool from non-reacting serum samples (low pool from all individuals prior to infection) were prepared and a checkerboard titration was performed by serial dilutions of the strongly reacting serum pool against dilutions of the detection antibody, anti-rabbit IgA (Abcam, USA) or anti-rabbit IgG (Southern Biotechnology, USA). Optimal dilutions for the serum and detector antibody were selected by visually identifying the inflection point from the resulting dilution curves; the dilutions established for the serum were 1:10 for IgA and 1:10,000 for IgG, while for anti-rabbit IgA it was determined to be 1:5,000 and for anti-rabbit IgG, 1:10,000. Each sample from each individual was performed in duplicate with all plates having the high, low and background controls. Serum samples from each rabbit at every sampling point were added to the wells in blocking buffer at the appropriate final dilutions, and incubated at $37^{\circ} \mathrm{C}$ for 2 hours in a humidified chamber. Plates were then washed 4 times with PBS-T between each incubation and developed with 2,2'-Azino-bis(3ethylbenzthiazoline-6-sulfonic acid) (Sigma-Aldrich) for 30 minutes and read with a spectrophotometer at 405 $\mathrm{nm}$. Values were expressed as immunosorbent optical densities (OD).

To confirm the consistency of the ELISA results among plates, the relationship between corrected high antibody controls (high control - background control) and corrected low antibody controls (low control - background control) was examined; plates were repeated if the ratio was not consistent with a linear relationship among all plates showing a Pearson's correlation coefficient above $r$ $=0.70$. Background corrected antibody values were then transformed and standardized into optical density (OD) indexes as: $\mathrm{Xi}=(\mathrm{OD}$ test sample-OD negative control)/ (OD positive control-OD negative control) where $\mathrm{Xi}$ represents a replicate for each individual at every sampling point; the average of the two replicates $(\mathrm{Xi}=$ mean $(\mathrm{X} 1+\mathrm{X} 2))$ was then estimated for each individual at each sampling point [39]. To remove the occasional negative values, data were corrected as: $\mathrm{Xi}^{*}=\mathrm{Xi}+\mid \min ($ total $\mathrm{Xi}) \mid$. The new standardized mean optical density indexes were used in the statistical analyses.

\section{Haematology}

A small aliquot $(0.2 \mathrm{ml})$ of blood collected weekly from every animal was stored in EDTA coated tubes (Sartorius, Germany) and the most common cellular components in the blood (neutrophils, lymphocytes, 
monocytes, eosinophils, basophils and red blood cells) were quantified using the Hemavet 3 hematology system (Drew Scientific, USA).

\section{Quantification of cytokines gene expression in the lung} Cytokine gene expression in the lung was determined using a real-time quantitative PCR approach. For RNA extraction and purification, tissues were homogenized with a rotor-stator (Polytron PT 2100, Kinematica) in TRIzol reagent (Invitrogen). RNA was digested with $4 \mathrm{U}$ TURBO DNA-free DNase (Ambion) and residual DNA contamination was assessed by performing quantitative PCRs. $1 \mu \mathrm{g}$ of RNA was reverse transcribed with the High Capacity RNA-to-cDNA kit (Applied Biosystems). The resultant complementary DNA product (cDNA) was used to detect expression of IFN- $\gamma$, IL-4 and IL-10, with Hypoxanthine Phosphoribosyl Transferase (HPRT) as the housekeeping gene [41], using commercially available or custom made primers and probes (Applied Biosystems). The primer and probe sequences were based on a plasmid kindly provided by Dr. Sheila Lukehart (University of Washington, WA) [41]. The plasmid was also used to verify amplification efficiencies of the primer probe pairs ( $450 \mu \mathrm{M}$ primers and $125 \mu \mathrm{M}$ probe) by performing quantitative PCRs with 10 -fold serial dilutions of the plasmid. The Pearson correlation coefficients between each cytokine and the housekeeping gene were always high (for all: $r>0.99$ ). The sequences of the primer-probe pairs were as follows: HPRT (forward primer; 5'-CTCTCAACCTTAACTGGAAAGAATGTCT-3', reverse primer; 5' - GGAAAGCAA GGTCTGCATTGTT-3', probe 5'-6FAM-TTGCCAGTG TCAATTAT-NFQ-3'), IFN- $\gamma$ (forward primer; 5'GCTTTTCAGCTCTGCCTCATCTT-3', reverse primer; 5'- GGTTAGTGTGTCCTGGCAGTAA-3', probe 5'6FAM-CAGCCGTAAGAACCC-NFQ-3'), IL-10 (Taqman assay ID; Oc03396942_m1, Applied Biosystems) and IL-4 (forward primer; $5^{\prime}$-ATGCACCAAGCTGATGATAGCA-3', reverse primer; 5'-CCTCTCTCTC GGTTGTGTTCTT-3', probe 5'-6FAM-CCCTGGCCGT CCCC-NFQ-3'). Quantitative PCRs were performed on an ABI7500 real time thermal cycler set in 'fast' mode for 40 cycles and using the PerfeCTa ${ }^{\mathrm{m}} \mathrm{qPCR}$ enzyme FastMix, UNG, Low ROX (Quanta Biosciences).

For visual purposes, changes in cytokines expression in the lungs were initially corrected for the house keeping gene (Ct values) and then scaled using the relative transcript level in fold change between infected at every sampling point (DPI) and the average of the controls from the whole experiment, following the comparative $2^{-\Delta \Delta C t}$ method [42]. For analytical purposes, the corrected $\mathrm{Ct}$ values were used.

\section{Data analysis}

Data were analyzed using linear mixed effect models (LME-REML) unless otherwise stated. To explore how bacteria shedding was affected by the host immune response, the number of colonies shed per interaction time was examined in relation to bacteria CFU count, antibody levels, blood cell values and infection time (week post infection WPI or days post infection DPI depending whether we used longitudinal or point based data). Individual identification code (ID) was considered as a random effect and the non-independent sampling of the same individual through time was quantified by including an autoregressive function of order 1 (AR1) on the individual ID. Changes in bacteria colonies established in the respiratory tract were examined in relation to the three respiratory organs and infection time (DPI), where individual ID was considered as a random effect and an autoregressive function of order 1 (AR1) was applied to the individual ID to take into account the non-independent response of the three correlated organs within each individual. This analysis was repeated for each organ and by including cytokines expression for the lungs. Linear mixed effect models were also performed to highlight differences between treatments (infected and control) and sampling time (WPI or DPI) in serum antibody response (IgA and $\operatorname{IgG}$ ), white blood cells concentration and cytokine expression; again the individual ID was treated as a random or correlated effect (AR1) when necessary.

\section{Abbreviations}

CFUs: Colony forming units; IgG: Immunoglobulin G; IgA: Immunoglobulin A; IL-10: Interleukin 10; IL-4: Interleukin 4; IFN- $\gamma$ : Interferon gamma; FHA:

filamentous haemagglutinin; DPI: Days post-infection; S.E.: Standard error; ID: Rabbit identification number; Ct: Cycle threshold; BG: Bordet-Gengou; OD: Optical density; SS: Stainer-Scholte; PBS: Phosphate buffered saline; PBS-T: PBS supplemented with $0.1 \%$ Tween-20; EDTA: Ethylene diamine tetraacetic acid; HPRT: Hypoxanthine Phosphoribosyl Transferase; ELISA: Enzyme Linked Immunosorbent Assay; AR1: Autoregressive function of order 1; LME-REML linear mixed model with restricted maximum likelihood.

\section{Acknowledgements}

We would like to thank E. Harvill and A. Hernandez for critical comments on the manuscript and Peter Hudson for pondering with IMC this study as part of a broader project on the immuno-epidemiology of co-infection. This work, AKP and KEC were funded by HFSP research grant.

\section{Author details}

${ }^{1}$ Center for Infectious Disease Dynamics, Dept. Biology, The Pennsylvania State University, University Park PA 16802, USA. ${ }^{2}$ Animal Resource Program, Centralized Biological Laboratory, The Pennsylvania State University, University Park PA 16802, USA.

\section{Authors' contributions}

AKP performed the animal experiment, undertook the lab analysis and discussed with IMC the original idea and design of the experiments. KEC worked as an undergraduate research assistant and helped with the animal and lab work, JRW assisted during the lab experiment and undertook the 
hematological analysis and IMC designed the experiment, helped with the animal experiment, carried out the analysis and their interpretation, conceived the paper and the original study. IMC and AKP wrote the paper with critical comments from JRW. All authors approved the final manuscript.

Received: 29 March 2010 Accepted: 25 August 2010

Published: 25 August 2010

\section{References}

1. Gupta S, Day KP: a theoretical framework for the immunoepidemiology of Plasmodium falciparum malaria. Parasite Immunol 1994, 16(7):361-370.

2. Hellriegel B: Immunoepidemiology - bridging the gap between immunology and epidemiology. Trends Parasitol 2001, 17(2):102-106.

3. Roberts MG: The immunoepidemiology of nematode parasites of farmed animals: A mathematical approach. Parasitol Today 1999, 15(6):246-251.

4. Woolhouse MEJ: A theoretical framework for the immunoepidemiology of helminth infection. Parasite Immunol 1992, 14(6):563-578.

5. Kaufmann $\mathrm{SH}$ : How can immunology contribute to the control of tuberculosis? Nat Rev Immunol 2001, 1(1):20-30.

6. Monack DM, Mueller A, Falkow S: Persistent bacterial infections: the interface of the pathogen and the host immune system. Nat Rev Microbiol 2004, 2(9):747-765.

7. Stewart GR, Robertson BD, Young DB: Tuberculosis: A problem with persistence. Nat Rev Microbiol 2003, 1(2):97-105.

8. Brockmeier SL, Lager KM: Experimental airborne transmission of porcine reproductive and respiratory syndrome virus and Bordetella bronchiseptica. Vet Microbiol 2002, 89(4):267-275.

9. Hermann JR, Brockmeier SL, Yoon KJ, Zimmerman JJ: Detection of respiratory pathogens in air samples from acutely infected pigs. Can J Vet Res 2008, 72(4):367-370.

10. Peek RM Jr, Blaser MJ: Helicobacter pylori and gastrointestinal tract adenocarcinomas. Nat Rev Cancer 2002, 2(1):28-37.

11. Saez-Llorens $X$, McCracken GH: Bacterial meningitis in children. Lancet 2003, 361(9375):2139-2148.

12. Stephens DS, Greenwood B, Brandtzaeg P: Epidemic meningitis, meningococcaemia, and Neisseria meningitidis. Lancet 2007, 369(9580):2196-2210.

13. Pathak AK, Boag B, Poss M, Harvill E, Cattadori IM: Seasonal incidence of Bordetella Bronchiseptica in an age-structured free-living rabbit population. Epidemiology and Infection

14. Cotter PA, Miller JF: BvgAS-mediated signal transduction - analysis of phase-locked regulatory mutants of Bordetella bronchiseptica in a rabbit model. Infect Immun 1994, 62(8):3381-3390.

15. Harvill ET, Cotter PA, Miller JF: Pregenomic comparative analysis between Bordetella bronchiseptica RB50 and Bordetella pertussis Tohama I in murine models of respiratory tract infection. Infect Immun 1999, 67(11):6109-6118.

16. Kirimanjeswara GS, Mann PB, Harvill ET: Role of antibodies in immunity to Bordetella infections. Infect Immun 2003, 71(4):1719-1724.

17. Pilione MR, Harvill ET: The Bordetella bronchiseptica type III secretion system inhibits gamma interferon production that is required for efficient antibody-mediated bacterial clearance. Infect Immun 2006, 74(2):1043-1049.

18. Pishko EJ, Kirimanjeswara GS, Pilione MR, Gopinathan L, Kennett MJ, Harvill ET: Antibody-mediated bacterial clearance from the lower respiratory tract of mice requires complement component C3. Eur J Immunol 2004, 34(1):184-193.

19. Stockbauer KE, Fuchslocher B, Miller JF, Cotter PA: Identification and characterization of BipA, a Bordetella Bvg-intermediate phase protein. Mol Microbiol 2001, 39(1):65-78.

20. Thakar J, Pilione M, Kirimanjeswara G, Harvill ET, Albert R: Modeling systems-level regulation of host immune responses. PLoS Comput Biol 2007, 3(6):e109.

21. Thakar J, Saadatpour-Moghaddam A, Harvill ET, Albert R: Constraint-based network model of pathogen-immune system interactions. J $R$ Soc Interface 2009, 6(36):599-612.

22. Irie $\mathrm{Y}, \mathrm{Yuk} \mathrm{MH}$ : In vivo colonization profile study of Bordetella bronchiseptica in the nasal cavity. FEMS Microbiol Lett 2007, 275(2):191-198.

23. Bemis DA, Shek WR, Clifford CB: Bordetella bronchiseptica infection of rats and mice. Comp Med 2003, 53(1):11-20.
24. Coers J, Starnbach MN, Howard JC: Modeling infectious disease in mice: co-adaptation and the role of host-specific IFN-gamma responses. PLOS Pathog 2009, 5(5)

25. Wolfe DN, Kirimanjeswara GS, Goebel EM, Harvill ET: Comparative role of Immunoglobulin A in protective immunity against the Bordetellae. Infect Immun 2007, 75(9):4416-4422.

26. Otten MA, van Egmond M: The Fc receptor for IgA (FcalphaRI, CD89). Immunol Lett 2004, 92(1-2):23-31.

27. Kirimanjeswara GS, Mann PB, Pilione M, Kennett MJ, Harvill ET: The complex mechanism of antibody-mediated clearance of Bordetella from the lungs requires TLR4. J Immunol 2005, 175(11):7504-7511.

28. Moore KW, de Waal Malefyt R, Coffman RL, O'Garra A: Interleukin-10 and the interleukin-10 receptor. Annu Rev Immunol 2001, 19:683-765.

29. O'Garra A, Vieira P: $\mathrm{T}(\mathrm{H}) 1$ cells control themselves by producing interleukin-10. Nat Rev Immunol 2007, 7(6):425-428.

30. Sukumar N, Love CF, Conover MS, Kock ND, Dubey P, Deora R: Active and passive immunizations with Bordetella colonization factor $A$ protect mice against respiratory challenge with Bordetella bronchiseptica. Infect Immun 2009, 77(2):885-895.

31. Naylor SW, Flockhart A, Nart P, Smith DG, Huntley J, Gally DL, Low JC: Shedding of Escherichia coli 0157:H7 in calves is reduced by prior colonization with the homologous strain. Appl Environ Microbiol 2007, 73(11):3765-3767.

32. Beagley KW, Timms P: Chlamydia trachomatis infection: incidence, health costs and prospects for vaccine development. J Reprod Immunol 2000, 48(1):47-68.

33. Taylor DN, Perlman DM, Echeverria PD, Lexomboon U, Blaser MJ: Campylobacter immunity and quantitative excretion rates in Thai children. J Infect Dis 1993, 168(3):754-758.

34. Ito Jl, Lyons JM: Role of gamma interferon in controlling murine chlamydial genital tract infection. Infect Immun 1999, 67(10):5518-5521.

35. Li W, Murthy AK, Guentzel MN, Seshu J, Forsthuber TG, Zhong G, Arulanandam BP: Antigen-specific CD4+ T cells produce sufficient IFNgamma to mediate robust protective immunity against genital Chlamydia muridarum infection. J Immunol 2008, 180(5):3375-3382.

36. Coutts AJ, Dawson S, Binns S, Hart CA, Gaskell CJ, Gaskell RM: Studies on natural transmission of Bordetella bronchiseptica in cats. Vet Microbiol 1996, 48(12):19-27.

37. Elahi S, Thompson DR, Strom S, O'Connor B, Babiuk LA, Gerdts V: Infection with Bordetella parapertussis but not Bordetella pertussis causes pertussislike disease in older pigs. J Infect Dis 2008, 198(3):384-392.

38. lemura R, Tsukatani R, Micallef MJ, Taneno A: Simultaneous analysis of the nasal shedding kinetics of field and vaccine strains of Bordetella bronchiseptica. Vet Rec 2009, 165(25):747-751.

39. Sanchez J, Dohoo IR, Markham F, Leslie K, Conboy G: Evaluation of the repeatability of a crude adult indirect Ostertagia ostertagi ELISA and methods of expressing test results. Vet Parasitol 2002, 109(1-2):75-90.

40. Crowther JR: The ELISA guidebook. Methods Mol Biol 2000, 149:III-IV, 1-413.

41. Godornes C, Leader BT, Molini BJ, Centurion-Lara A, Lukehart SA: Quantitation of rabbit cytokine mRNA by real-time RT-PCR. Cytokine 2007, 38(1):1-7.

42. Schmittgen TD: Analysis of Relative Gene Expression Data Using RealTime Quantitative PCR and the $2^{-\Delta \Delta C}$ T Method. Methods 2001, 25(4):402-408

doi:10.1186/1471-2180-10-226

Cite this article as: Pathak et al: Immune regulation of a chronic bacteria infection and consequences for pathogen transmission. BMC Microbiology 2010 10:226. 